\title{
Zinc Finger and BTB Domain Containing Protein 7A
}

National Cancer Institute

\section{Source}

National Cancer Institute. Zinc Finger and BTB Domain Containing Protein 7A. NCI

Thesaurus. Code 143387.

Zinc finger and BTB domain-containing protein 7A (584 aa, $\sim 61 \mathrm{kDa}$ ) is encoded by the human ZBTB7A gene. This protein plays a role in the regulation of both transcription and B-cell development. 